\title{
Up-Regulation of Mir-105-5p Promotes The Development of Breast Cancer By Targeting AKT1 / GRB2 Genes In Patients With Breast Cancer
}

\section{Fatemeh Rezaei}

Islamic Azad University

Flora Forouzesh ( $\square$ f8forouzesh@gmail.com )

Islamic Azad University https://orcid.org/0000-0002-2747-3479

\section{Fereshteh Abbasvandi}

Motamed Cancer Institute

\section{Research Article}

Keywords: Breast cancer, miR-105-5P, AKT1 pathway, GRB2 gene

Posted Date: February 1st, 2022

DOI: https://doi.org/10.21203/rs.3.rs-1260114/v1

License: (c) (i) This work is licensed under a Creative Commons Attribution 4.0 International License.

Read Full License 


\section{Abstract}

Objective: miR-105-5p either acts as an oncomiR or tumor suppressor that has been shown to have various expression levels in a wide range of diseases and targets RAC-alpha serine/threonine-protein kinase (AKT1) and Growth Factor Receptor-bound protein 2 (GRB2) genes. GRB2 is a signaling protein that takes part in various signaling pathways. Due to the fact that miRNAs by acting on target genes, and regulates the expression of them, so the aim of this study is to evaluate the expressions of miR-105-5P, $A K T 1$, and $G R B 2$ genes and the correlation between them in breast cancer (BC) tissue compared to normal-adjacent tissues (NATs) in women with breast cancer.

Materials and methods: We utilized bioinformatics analysis to searching for target genes of miR-105-5p. Then, $35 \mathrm{BC}$ and NATs tissues were taken. In this experimental study, quantitative Real-Time PCR was performed to evaluate miR-105-5P, AKT1 and GRB2 genes expression, and the correlation between them was evaluated. The relation between expression and features of clinicopathological was explored.

Results: The miR-105-5p expression was increased significantly in BC tissues in comparison to NATs (P 0.05). The expression levels of $A K T 1$ and GRB2 genes were decreased significantly in $B C$ tissue in comparison to NATs (P 0.05). An inverse correlation was observed between miR-105-5p, AKT1 and GRB2 expression which was not significant $(\mathrm{P}>0.05)$. Also, a direct correlation was observed between $G R B 2$ and AKT1 gene expression (P 0.05).

Conclusion: Our findings revealed that increased expression levels of miR-105-5p in patients with breast cancer caused a decrease in the expression level of its target genes, AKT1 and GRB2, and subsequently lead to tumor progression and invasion of the tumor. miR-105-5p may serve as a promising target for BC therapy.

\section{Introduction}

Breast cancer (BC) is widely assumed to be the main cause of cancer death in women around the world. Every year, 2,088,849 new cases are diagnosed with BC with over 626,679 deaths estimated worldwide [1]. It is a multifactorial disorder associated with the effects of multiple genes in combination with environmental factors $[2,3,4]$. $B C$ is classified into two forms of sporadic $B C$, which approximately attributes to 95 percent of $\mathrm{BCs}$, and familiar $\mathrm{BC}$ which accounts for the rest of the BCs and it is caused by mutations in genes like Breast cancer susceptibility gene 1 (BRCA1) and Breast cancer susceptibility gene 2 (BRCA2)[5]. There are five major molecular subtypes of $\mathrm{BC}$. They are grouped based on the genes expressed: Luminal A BC is hormone-receptor positive (progesterone-receptor positive and/or estrogenreceptor), HER2 negative, and the protein $\mathrm{Ki}-67$ is in low levels. Luminal B BC is hormone-receptor positive (progesterone-receptor positive and/or estrogen-receptor), and either HER2 positive or HER2 negative with high levels of Ki-67. Triple-negative/basal-like BC is HER2 negative and hormone-receptor negative (progesterone-receptor and estrogen-receptor negative. HER2-enriched BC is HER2 positive and hormonereceptor negative (progesterone-receptor and estrogen-receptor negative). Normal-like BC is similar to 
luminal A disease: HER2 negative and hormone-receptor positive (progesterone-receptor and/or estrogenreceptor positive), and the protein $\mathrm{Ki}-67$ is in low levels [6].

In the past decade, with the usage of genome-wide studies on DNA sequence, gene expression, and so forth, many aberrant genes and miRNAs have been identified which might contribute to breast tumor [7, 8]. miR-105-5P targets many genes in various cancers and can act either as tumor suppressor or oncomiR [9]. Some studies showed that miR-1 to be associated with several diseases such as cancer (BC, human gliomas, colorectal cancer, gastric cancer, prostate cancer and hepatocellular carcinoma) and heart diseases [9]. miR-105 functions as an oncogene in several of these cancers such as colorectal cancer and BC, and high expression of this miR causes metastasis and invasion. But in cancers such as prostate cancer, hepatocellular carcinoma, human gliomas, and lung cancer, miR-105 functions as a tumor suppressor. miR-105 can alter the expression levels of its target gene, so it has an impact on cancer progression [9]. miR-105-5P is secreted by cancer cells and in BC, it works as oncomiR via targeting Z01 protein, which acts as a tight junction adaptor protein, and therefore, it contributes to metastasis . miR-105-5P targets when this micro RNA is suppressed in BCs it can lead to a decrease in the metastasis rate $[10,11]$.

Other genes such as $A K T 1$ which are involved in different cell signaling pathways and any dysregulation of their expression may lead to creation of cancer cells [12].

AKT1 is serine-threonine protein kinase which regulates numerous processes such as proliferation, cell survival, growth, and so on $[13,14]$. AKT1 is a mediator of various cellular pathways including $P I 3 K /$ PTEN / AKT / mTORC1, therefore, it has distinct roles in the development of $\mathrm{BC}[15,16]$. It has been assumed that overexpression and activation of $A K T 1$ might lead to $\mathrm{BC}$ progression by contributing to being resistant to anti-proliferative signals $[17,18]$.

Growth Factor Receptor-bound protein 2 (GRB2) gene is an adapter protein that plays an important link between cell surface growth factor receptors and the Ras signaling pathway and it may have a vital role in cell survival, proliferation, differentiation and angiogenesis [19]. It has been reported that GRB2 is involved in development and progression of various malignancies such as BC, lung cancer, gastric cancer, colorectal cancer and so forth $[20,21,22]$. The aim of the current study is to evaluate the expression of miR-105-5p, AKT1, and GRB2 genes and the correlation between them in BC tissues from Iranian patients with $\mathrm{BC}$ and adjacent normal tissue (NATs) from same patients.

\section{Material And Methods}

\section{Subjects}

In this experimental study, fresh breast cancer (BC) tissues and paired normal adjacent tissues (NATs) (>5 $\mathrm{cm}$ from tumor) from 35 women with BC were collected from Imam Khomeini Hospital, Tehran, Iran. Tissues were carried to the laboratory via liquid nitrogen tank. The BC patients had the average age of 50 years when diagnosed (range 30-55 years). Patients did not undergo any radiation therapy or 
chemotherapy. The diagnosis, histological grade and the clinical stage of each case were independently confirmed by a pathologist. Patients had no other diseases and the ones that did, were excluded from the study. The study was approved by the Ethics Committee and all of the contributors provided written informed consent.

\section{RNA isolation and cDNA synthesis}

Total RNA was extracted from the fresh BC tissues using XS spin kit (Macherey-Nagel, Germany, LOT No: 1301/005), based on the manufacturer's instruction. After applying DNase-I for removing DNA contamination, complementary DNA strand (CDNA) was synthesized using the PrimeScript ${ }^{\text {TM }}$ RT Rreagent Kit (Takara, Japan, Cat No: RR037A) according to the manufacturer's protocol. For evaluation of miR-105$5 p$ expression, stem loop cDNA was synthesized. The RNA samples were evaluated on Nanodrop 2000 spectrophotometer (Thermo Scientific, Wilmington, DE, USA) for both quantity and also quality

Target genes prediction of miR-105-5p

miRNA targets were predicted and determined using the algorithms of TargetScan 7.1 [23], miRBase [24], miRanda [25], miRDIP 4.1 [26], miRWALK 3.0 [27]and miRDB 5.0 [28].

\section{Real-time quantitative PCR (qPCR)}

Quantitative Real-time PCR was carried out using ABI 7900HT instrument (ABI Inc.), WizPure ${ }^{\text {TM }}$ qPCR Master (SYBR) (Korea, Cat No: w1711). U6 snRNA was used for miR-105-5p normalization. Specific primers were brought as follow: U6 forward: 5'- GCTTCGGCAGCACATATAC -3', and U6 reverse: 5'ATTCCGTTTCTGGGAGGG-3', GRB2 forward: 5'-ATTCCTGCGGGACATAGAACA -3', GRB2 reverse: 5'AGTTCCAACCAAAGTGAGAGGG-3', AKT1 forward: 5'-TCTATGGCGCTGAGATTGTG -3', AKT1 reverse: 5' CTTAATGTGCCCGTCCTTGT -3', miR-105-5p forward: 5' - TCAAATGCTCAGACTCCTGTGGTGTCGT-3' and miR-105-5p reverse: 5 '- CCAGTGCAGGGTCCGAGGTA-3. The qPCR reaction for AKT1 and GRB2 genes and miR-105-5p were performed with the following reaction: $20 \mu \mathrm{L}$ volume reaction containing $1 \mu \mathrm{l}$ of template cDNA (12.5 ng/ $\mu \mathrm{L}), 0.5 \mu$ l of each $10 \mathrm{pmol} / \mathrm{ul}$ primer (Sinaclone, Iran), $10 \mu$ l of SYBR Green PCR master mix and $8 \mu \mathrm{dH}_{2} \mathrm{O}$ and followed by initial denaturation at $95^{\circ} \mathrm{C}$ for $5 \mathrm{~min}$, followed by 40 cycles of denaturation at $95^{\circ} \mathrm{C}$ for 30 secs, annealing at $60^{\circ} \mathrm{C}$ for 30 secs. GAPDH gene was used for AKT1 and GRB2 genes for normalization. GAPDH forward (F): 5'- ATTTGGTCGTATTGGGCG -3' and GAPDH reverse (R): $5^{\prime}$ - GTACTCAGCGCCAGCATC $-3^{\prime}$. The relative gene expression level was assessed by using $2^{-\triangle \triangle C t}$ quantitative method (29), each sample was analyzed in duplicate.

\section{Statistical analysis}

All experimental information was expressed as mean \pm SEM. Statistical comparisons between the BC tissues and NATs were carried out using paired t-test and one-way analysis of variance was carried out to compare the differences among more than two groups followed by Tukey's test. Differences were considered statistically significant at $\mathrm{P} \leq 0.05$. The correlation between $A K T 1$, and GRB2 genes and miR- 
105-5p in BC patients was assessed by Pearson's correlation. In this study, GraphPad Prism was used to carry out all statistical analyses (GraphPad Software, La Jolla California USA).

\section{Results}

The clinicopathological characteristics of patients are presented in Table 1.

Table 1. Clinicopathologic characteristics of patients with BC

\begin{tabular}{|lll|}
\hline Characteristics & Number of patients & Number analyzed (\%) \\
\hline Age at disease onset (year) & \\
\hline & 19 & $53.9 \%$ \\
& 16 & $46.1 \%$ \\
\hline Clinical Stage & & \\
\hline stage I & 2 & $5.6 \%$ \\
\hline stage II & 24 & $67.2 \%$ \\
\hline stage III & 9 & $27.2 \%$ \\
\hline Histological grade & & $14 \%$ \\
Grade- I & 5 & $67.2 \%$ \\
\hline Grade- II & 24 & $16.8 \%$ \\
Grade- III & 6 & \\
\hline
\end{tabular}

\section{miR-105-5p is upregulated in Breast cancer}

Quantitative real-time PCR was used to evaluate the expression levels of miR-105-5p in breast cancer (BC) and paired normal adjacent tissues (NATs). We observed that there was a significant up-regulated in $B C$ samples relative to that in NATs as the control group (Fig. 1) ( $p=0.0194)$. This finding indicates that $m i R-105-5 p$ may be a regulator of molecular mechanisms in BC progression. The relationship between 
miR-105-5p expression and clinicopathological characteristics of BC patients including histological grade, clinical stages and patients' age were examined. An increase in the expression level of miR-105$5 p$ was observed in stage I and grade I compared with other grade (II - III) and stage (II-III). BC patients were divided according to age into the younger and older groups (women aged $\leq 50$ and $>50$ years, respectively). In older women (aged $>50$ years) miR-105-5p expression was increased compared with younger women (aged $\leq 50$ years) but no difference was found in the rate of miR-105-5p mRNA expression in according to age. In our study, statistical analysis showed that the miR-105-5p expression was not significantly related to clinicopathological features including histological grade, clinical stages and patients' age $(p>0.05)$ (Table 2).

Table 2: Correlation between miR-105-5p expression and Clinicopathological Characteristics in Breast Cancer patients

\begin{tabular}{|lcc|}
\hline Characteristics & SE of difference & P value \\
\hline Age at disease onset (year) & & \\
\hline$<50$ vs $\geq 50$ & 0.2952 & 0.5777 \\
\hline Histological grade & & \\
& & \\
Grade I vs. Grade II & 0.4478 & 0.8908 \\
Grade I vs. Grade III & 0.5515 & 0.5352 \\
Grade II vs. Grade III & 0.4157 & 0.6231 \\
& & \\
Breast cancer staging & & \\
Stage I vs. Stage II & 0.7149 & 0.0004 \\
Stage I vs. Stage III & 0.7593 & $<0.0001$ \\
Stage II vs. Stage III & 0.3796 & 0.2733 \\
& & \\
\hline
\end{tabular}

\section{Prediction of target genes of $m i R-105-5 p$}

Various target prediction programs (TargetScan,_miRBase, miRanda, mirDIP, miRWALK, and miRDB) were used to explore the potential mechanism and analyze the AKT1 and GRB2 target genes of miR-105$5 p$ (ACESSION: MIMAT0000102). Based on the predicted genes, score class of $A K T 1$ gene is top up $5 \%$ as high-throughput miR-105-5p targeting and Score class of GRB2 gene is top up $1 \%$ as very highthroughput miR-105-5p targeting. 


\section{$A K T 1$ is downregulated in Breast cancer}

We first investigated the expression of $A K T 1$ in $\mathrm{BC}$ tissues and NATs by quantitative RT-PCR. We observed the expression levels of $A K T 1$ was decreased in $\mathrm{BC}$ tissues compared to that in NATs significantly (Fig. 2) ( $p=0.0141$ ). Next, we compared the expression levels of $A K T 1$ with the clinicopathological features including histological grade, clinical stages and patients' age. The lowest expression of $A K T 1$ was observed in Stage III and grade I. But, the AKT1 gene expression was not significantly related to clinicopathological features including histological grade, and clinical stages.

To better understand the relationship between AKT1 expression and patients' age, we considered the expression of this gene in younger and older groups ( $\leq 50$ year and $>50$ year). No significant difference was observed between aged $\leq 50$ year and aged $>50$-year groups $(P=0.680)$. However, there was a trend toward decreasing $A K T 1$ expression in aged $>50$-year groups (Table 3 ).

Table 3: Correlation between AKT1 expression and Clinicopathological Characteristics in Breast Cancer Patients

\begin{tabular}{|lcc|}
\hline Characteristics & SE of difference & P value \\
\hline Age at disease onset (year) & & \\
\hline$<50$ vs $\geq 50$ & 0.6882 & 0.6808 \\
\hline Histological grade & & \\
& & \\
Grade I vs. Grade II & 0.6956 & 0.5266 \\
Grade I vs. Grade III & 0.8569 & 0.9801 \\
Grade II vs. Grade III & 0.6459 & 0.6311 \\
& & \\
\hline Breast cancer staging & & \\
\hline Stage I vs. Stage II & 1.621 & 0.3079 \\
Stage I vs. Stage III & 1.722 & 0.1682 \\
Stage II vs. Stage III & 0.8608 & 0.6434 \\
\hline
\end{tabular}

\section{$A K T 1$ is a target of $m i R-105-5 p$}

Bioinformatics prediction revealed potential binding sites of miR-105-5p on the 3'-untranslated region (3'UTR) of AKT1 (Fig. 3a). Spearman's rank order correlation was used to determine the relation between 
miR-105-5p expression and its target gene. We observed a negative correlation between miR-105-5p and AKT1 in $\mathrm{BC}$ tissues (slope $=-0.008862, \mathrm{P}=0.9602$ ) but not significantly (Fig. $3 \mathrm{~b}$ ). In the other hand, the expression of miR-105-5p was up-regulated, whereas $A K T 1$ was downregulated in the tumor tissues. These findings collectively indicate that $A K T 1$ is a target of miR-105-5p in BC.

\section{GRB2 is downregulated in Breast cancer}

The expression level GRB2 was quantified by quantitative real-time PCR. We observed that there was a significant decrease in GRB2 expression in BC tissue samples in comparison to NATs ( $p=0.0473$ ) (Fig .4). We next explored the relationship between GRB2 and clinicopathological characteristics of BC patients. The lowest expression of GRB2 was observed in Stage III and grade I. But the significant difference was not found when the gene expression was compared between different histological grades (I to III) and different clinical stages (I to III). Also, the expression of GRB2 in patients aged > 50-year groups was lower than aged $<50$ year, but no correlation was observed between $G R B 2$ expression and age ( $<50$ year and $>$ 50-year groups) $(P=0.585)$ (Table 4).

Table 4: Correlation between GRB2 expression and Clinicopathological Characteristics in Breast Cancer Patients

\begin{tabular}{|lcc|}
\hline Characteristics & SE of difference & P value \\
\hline Age at disease onset (year) & & \\
\hline$<50$ vs $\geq 50$ & 4.104 & 0.5853 \\
\hline Histological grade & & \\
& & \\
Grade I vs. Grade II & 7.184 & 0.9198 \\
Grade I vs. Grade III & 8.849 & 0.3992 \\
Grade II vs. Grade III & 6.670 & 0.3946 \\
& & \\
Breast cancer staging & & \\
\hline Stage I vs. Stage II & 10.11 & 0.5194 \\
Stage I vs. Stage III & 10.74 & 0.2622 \\
Stage II vs. Stage III & 5.368 & 0.5123 \\
\hline
\end{tabular}


Bioinformatics prediction indicated that GRB2 is the potential target gene of $m i R-105-5 p$. The 3'-UTR of GRB2 mRNA has a specific sequence which is complementary to the seed region of miR-105-5p (Fig. $5 a)$

We analyzed $35 \mathrm{BC}$ tissues and NATs to explore the relationship between miR-105-5p and the target GRB2 gene. The Spearman's correlation demonstrated that miR-105-5p expression had negative correlation with the GRB2 expression (slope $=-0.02614, \mathrm{p}=0.4563$ ) but not significantly shown in (Fig. $5 b)$. Showed miR-105-5p was up-regulated in BC tissues while GRB2 was down-regulated in BC tissues.

Moreover, we explored the relationship between two target genes, AKT1 gene and GRB2 gene. We observed a significant positive correlation between $A K T 1$ gene and $G R B 2$ gene (slope $=3.582, \mathrm{p}<0.0001$ ) in $\mathrm{BC}$ tissues. This finding indicates that AKT1 gene and GRB2 gene can be a regulator of signaling pathway in $\mathrm{BC}$ progression.

\section{Discussion}

Our study indicated that miR-105-5p expression was up-regulated in BC tissues in comparison to NATs. that it was statistically significant. In some studies, it was observed that miR-105-5p acts as tumor suppressor. Honeywell et al. and Liu et al. found out that miR-105-5p inhibits prostate tumor growth and human glioma cell progression respectively and its reduced expression can lead to these types of cancers $[10,30]$. The same result was found with non-small cell lung cancer [31]. It was also revealed that in some studies, miR-105-5p acts as onco-miR. miR-105-5p expression level was increased in gastric cancer cells and colorectal cancer cells $[9,32]$. Our results indicated miR-105-5p acts as onco-miR in BC tissue because the expression level of miR-105-5p is increased in BC tissue compared with NATs, and it is revealed that miR-105-5p may be relevant with cell proliferation. miRNAs have been known to have a role in regulating most biological processes and therefore, they can be used in order to gain an insight into the complex processes, including cell proliferation in cancer. Our bioinformatics studies showed that miR105-5p targets AKT1 pathway and GRB2 gene. AKT signaling pathway has been a major signaling node within the cells [33]. AKT1 pathway dysregulation could possibly lead to disruption of the balance between survival and death and it can also have a major impact on cancer development and therapy. Our results demonstrated that $A K T 1$ expression level significantly decreased in $\mathrm{BC}$ tissue compared with NATs even the expression of this gene is more in the advanced stages of BC tissues. Our results agreement with previous studies. Heng et al. found out that $A K T 1$ expression level was significantly lowered in breast tumor in comparison to matched adjacent normal tissues [34]. We also observed miR105-5p had negative correlation with expression of AKT1 in BC tissues. This result can be justified by Shen et al. 's finding in which they demonstrated that miR-105-5p can inhibit PI3K/AKT pathway in hepatocellular cancer [12].

GRB2 is an essential signaling mediator in various oncogenic signaling pathways and it has role-playing in several human malignancies [35]. The GRB2 has a wide association with a number of irregularities. So, it is an important target for the design of remedial anticancer strategies [36]. Lin-Yan et al. found that 
overexpression of GRB2 had correlation lymph node metastasis [37]. Yu et al. demonstrated that GRB2 is over expressed in gastric cancer [22]. Watanabe et al. found that GRB2 expression was significantly increased in human bladder cancer cell lines [38]. Lim et al. found out that GRB2 downregulation led to AKT inactivation and consequently it caused BC [38]. Our results revealed that GRB2 expression level significantly decreased in BC tissue compared with NATs. Its expression level lowered more in the advanced stages of BC. We demonstrated that miR-105-5p negatively modulated GRB2 by combining with the 3'-UTR of GRB2 gene.

Furthermore, another important aspect of this study showed that strong positively correlated between the expression levels of $A K T 1$ and GRB2 genes. So, that downregulation of GRB2 genes suppressed the activation of $A K T 1$ pathway. Lim et al. found that downregulation of GRB2 can lead to AKT1 inactivation and subsequently can cause lowered expression of $A K T 1$ [39]. These findings support the pivotal role of AKT pathway in modulating the BC development. Based on our results, we speculate that miR-105-5p may contribute to the development of $\mathrm{BC}$ via downregulation of $A K T 1$ and GRB2 genes.

miR-105 affects the genes which are involved in intracellular signaling pathways and this leads to the downregulation of GRB2 and GRB2 downregulates $A K T 1$ through p13k signaling pathway. Consequently, these alterations lead to more invasion and metastasis in BC.

\section{Conclusion}

Our findings demonstrated miR-105-5p was up-regulated in human BC. miR-105-5p promotes cell proliferation in $\mathrm{BC}$ by targeting $A K T 1$ and $G R B 2$ genes. Up-regulation of miR-105-5p could promote $\mathrm{BC}$ growth by decreasing the activity of AKT1 signaling pathways and GRB2.

Our Insilco analyses showed that $A K T 1$ and GRB2 are the direct targets of miR-105-5p and basically micro RNAs effect on the cell through regulating their target genes. Increased expression of miR-105-5p downregulate the expression of GRB2 and $A K T 1$ and therefore these changes cause tumor progression and tumor invasion. These two genes are important genes in intracellular signaling pathways and reduced expression of them can dysregulate the cell cycle and cause tumor formation. Therefore, identifying a specific biomarker, and gaining an understanding into the underlying mechanism might provide feasible therapeutic approaches to overcome cancer. By targeting miR-105-5p, it might be considered as a possible therapeutic strategy for $\mathrm{BC}$ treatment. However, the detailed mechanism of miR105-5p in BC progression needs further investigations.

\section{Declarations}

\section{Conflict interest}

The authors declare no conflict of interests in this study.

\section{Authors' contributions}


F.F. Participated in study design. F.R. and F.A. collected the samples. F.F, F.R. and F.A. participated in data collection and evaluation, drafting. F.F and F.R. participated in RT-qPCR analysis and statistical analysis. F.R. prepared lab working, and doing molecular experiments. F.F. and F.R. Contributed extensively in the interpretation of the data and the conclusion. All authors performed editing and approving the final version of this paper for submission, also participated in the finalization of the manuscript, and approved the final draft.

\section{Acknowledgments}

This paper is based on MSc thesis of Fatemeh Rezaei, Faculty of Advanced Science and Technology, Tehran Medical Sciences, Islamic Azad University, Tehran, Iran.

\section{Ethical issues}

Approval for the study was obtained from the Ethics Committee of Islamic Azad Tehran Medical Sciences University-Pharmacy and Pharmaceutical Branches Faculty. Ethical code is IR.IAU.PS.REC.1398.069.

\section{References}

1- Bray F, Ferlay J, Soerjomataram I, Siegel RL, Torre LA, Jmal A. Global cancer statistics 2018 : GLOBOCAN estimates of incidence and mortality worldwide for 36 cancers in 185 countries . CA. 2018;68(6):394-424.

2- Pharoah PD, Dunning AM, Ponder BA, Ponder BA, Easton DF. Association Studies for Finding CancerSusceptibility Genetic Variants. NAT. 2004;4(11):850-860.

3- Blackburn GL, Wang KA. Dietary Fat Reduction and Breast Cancer Outcome: Results from the Women's Intervention Nutrition Study (WINS). Am J CLIN NUTR. 2007;86(3):878-881.

4- Kaiser J. Cholesterol Forges Link between Obesity and Breast Cancer. AAAS. 2013; 342(6162):1028

5- Garber JE, Offit K. Hereditary Cancer Predisposition Syndromes. AM. J Clin. Oncol. 2005;23(2):276292.

6- Al-thoubaity FKH. Molecular classification of breast cancer: A retrospective cohort study. AMS. 2019;49:44-48

7- Gray J, Druker B. The Breast Cancer Landscape. Nature. 2012; 486(7403): 328-329.

8- Stephens PJ, Tarpey PS, Davies H, Van Loo P, Greenman C, Wedge DC, et al. The Landscape of Cancer Genes and Mutational Processes in Breast Cancer. Nature. 2012;486(7403):400-404.

9- Li J, Zhang Z, Chen F, Hu T, Peng W, Gu Q, et al. The Diverse Oncogenic and Tumor Suppressor Roles of MicroRNA-105 in Cancer. FRONT ONCOL. 2019. 
10- Honeywell DR, Cabrita MA, Zhao H, Dimitroulakos J, Addison CL. MiR-105 Inhibits Prostate Tumour Growth by Suppressing CDK6 Levels. PloS One. 2013;8(8):70515.

11-Zhou W, Fong MY, Min Y, Somlo G, Liu L, Palomares MR, et al. Cancer-Secreted MiR-105 Destroys Vascular Endothelial Barriers to Promote Metastasis. Cancer Cell. 2014;25(4):501-515.

12-Shen G, Rong X, Zhao J, Yang X, Li H, Jiang H, et al. MicroRNA-105 Suppresses Cell Proliferation and Inhibits PI3K/AKT Signaling in Human Hepatocellular Carcinoma. Carcinogenesis. 2014;35(12):27482755.

13- Manning BD, Cantley LC. AKT/PKB Signaling: Navigating Downstream. Cell. 2007;129(7):12611274.

14- Yoeli-Lerner M, Toker A. Akt/PKB Signaling in Cancer: A Function in Cell Motility and Invasion. Cell Cycle. 2006;5(6):603-605.

15- Davis NM, Sokolosky M, Stadelman K, Abrams SL, Libra M, Candido S, et al. Deregulation of the EGFR/PI3K/PTEN/Akt/MTORC1 Pathway in Breast Cancer. Possibilities for Therapeutic Intervention. Oncotarget. 2014;5(13):4603-4650.

16- Huemer F, Bartsch R, Gnant M. The PI3K/AKT/MTOR Signaling Pathway: The Role of PI3K and AKT Inhibitors in Breast Cancer. Curr. Breast Cancer Rep. 2014;6:59-70.

17- Chin RY, Toker A. The Actin-Bundling Protein Palladin Is an Akt1-Specific Substrate That Regulates Breast Cancer Cell Migration. Molecular Cell. 2010;38(3):333-344.

18- Liang J, Zubovitz J, Petrocelli T, Kotchetkov R, Connor MK, Han K, et al. PKB/Akt Phosphorylates P27 Impairs Nuclear Import of P27 and Opposes p-27 Mediates G1 Arrest. Nature Medicine. 2002;8:11531160.

19- Ward AC, Smith L, De Koning JP, van Aesch Y, Touw IPT. Multiple Signals Mediate Proliferation, Differentiation, and Survival from the Granulocyte Colony-Stimulating Factor Receptor in Myeloid 32D Cells. J B C. 1999;274(21):14956-14962.

20- Pendergast AM, Quilliam LA, Cripe LD, Bassing CH, Dai Z, Li N, et al. BCR-ABL-Induced Oncogenesis Is Mediated by Direct Interaction with the SH2 Domain of the GRB-2 Adaptor Protein. Cell. 1993;75(1):175-185.

21- Timsah Z, Berrout J, Suraokar M, Behrens C, Song J, Lee JJ, et al. Expression Pattern of FGFR2, Grb2 and Plcy1 Acts as a Novel Prognostic Marker of Recurrence Recurrence-Free Survival in Lung Adenocarcinoma. Am. J Cancer Res. 2015;5(10):3135.

22- Yu GZ, Chen Y, Wang JJ. Overexpression of Grb2/HER2 Signaling in Chinese Gastric Cancer: Their Relationship with Clinicopathological Parameters and Prognostic Significance. J. Cancer Res. Clin. Onco. 
2009;135:1331-1339.

23-Riffo-Campos AL, Riquelme I, Brebi-Mieville P. Tools for Sequence-Based MiRNA Target Prediction: What to Choose? I J M S. 2016;17(12):1987.

24- Kozomara A, Birgaoanu M, Griffiths-Jones S. MiRBase: From MicroRNA Sequences to Function. Nucleic Acids Res. 2019;47(D1):D155-D162.

25-Zhao B, Xue B. Significant Improvement of MiRNA Target Prediction Accuracy in Large Datasets Using Meta-Strategy Based on Comprehensive Voting and Artificial Neural Networks. BMC Genomics. 2019;20(1):158.

26- Tokar T, Pastrello Ch, Rossos AEM, Abovsky M, Hauschild AC, Tsay M, et al. MirDIP 4.1-Integrative Database of Human MicroRNA Target Predictions. Nucleic Acids Res. 2018;46(D1):360-370.

27- Sticht C, Torre CDL, Parveen A, Gretz N. MiRWalk: An Online Resource for Prediction of MicroRNA Binding Sites. PloS One. 2018;13(10):0206239.

28- Chen Y, Wang X. MiRDB: An Online Database for Prediction of Functional MicroRNA Targets. Nucleic Acids Res. 2020;48(D1):127-131. .

29- Livak KJ, Schmittgen TD. Analysis of Relative Gene Expression Data Using Real-Time Quantitative PCR and the 2- $\Delta \Delta$ CT Method. Methods. 2001;25(4):402-408.

30- Liu X, Wang H, Zhu Z, Ye Y, Mao H, Zhang S. Micro RNA-105 Targets SOX 9 and Inhibits Human Glioma Cell Progression. FEBS Letters. 2016;590(23):4329-4342.

31- Lu G, Fu D, Jia C, Chai L, Han Y, Liu J, et al. Reduced MiR-105-1 Levels Are Associated with Poor Survival of Patients with Non-small Cell Lung Cancer. Onco. Lett. 2017;14(6):7842-7848.

32- Liu D, Hu X, Zhou H, Shi G, Wu J. Identification of Aberrantly Expressed MiRNAs in Gastric Cancer. GASTROENT RES PRACT. 2014. 2014;2014:1-9.

33- Liu P, Cheng H, Roberts T M, Zhao JJ. Targeting the Phosphoinositide 3-Kinase Pathway in Cancer. Nat. Rev. Drug Discov. 2009;8:627-644.

34. Heng J, Guo X, Wu W, Wang Y, Li G, Chen M, et al. Integrated Analysis of Promoter Mutation, Methylation and Expression of AKT1 Gene in Chinese Breast Cancer Patients. PloS One. 2017;12(3):114.

35- Giubellino A, Burke TR, Bottaro DP. Grb2 Signaling in Cell Motility and Cancer. Expert Opin. Ther. 2008;12(8):1021-1033.

36- ljaz M, Shahabz M, Jiang W, Fathy AH, Nesa EU, Wang D, et al. Oncogenic Role of Grb2 in Breast Cancer and Grb2 Antagonists as Therapeutic Drugs. Canc Therapy \& Oncol Int J. 2017;3(4):1-7. 
37- Li LY, Li EM, Wu ZY, Cao HH, Shen JH, Xu XE, et al. Overexpression of GRB2 Is Correlated with Lymph Node Metastasis and Poor Prognosis in Esophageal Squamous Cell Carcinoma. Int. J. Clin. Exp. Pathol. 2014;7(6):3132-3140.

38- Watanabe T, Shinohara N, Moriya K, Sazawa A, Kobayashi Y, Ogiso Y, et al. Significance of the Grb2 and Son of Sevenless (Sos) Proteins in Human Bladder Cancer Cell Lines. IUBMB Life. 2000;49(4):317320.

39- Lim SJ, Lopez-Berestein G, Hung MC, Lupu R, Tari AM. “Grb2 Downregulation Leads to Akt Inactivation in Heregulin-Stimulated and ErbB2-Overexpressing Breast Cancer Cells. Oncogene. 2000;19(54):6271-6276.

\section{Figures}

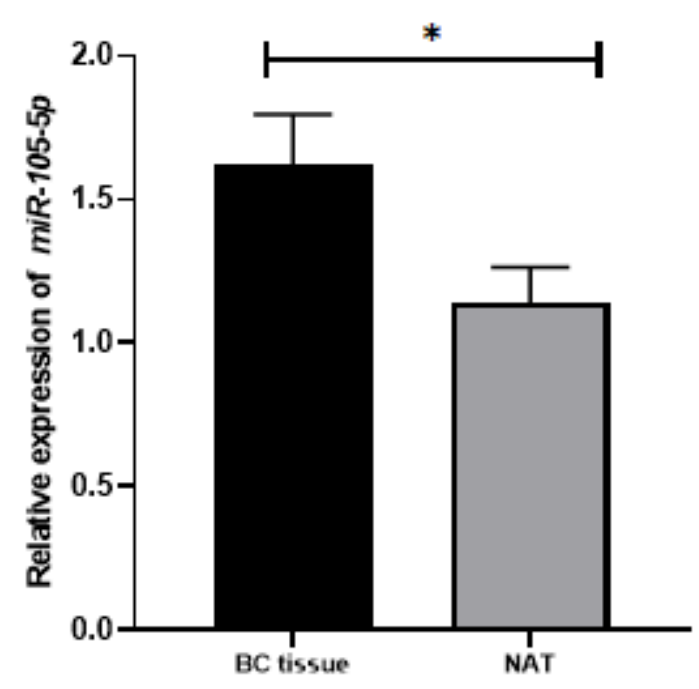

Figure 1

miR-105-5p is up-regulated in BC tissues and NATs. Relative miR-105-5p expression levels in BC tissues and NATs were determined by qRT-PCR. The expression of miR-105-5p is up-regulated in BC tissues compared with NATs ( $\left.{ }^{*} p 0.05\right)$. U6snRNA was used as an internal control. Abbreviation: BC: breast cancer, NATs: paired normal adjacent tissues. 


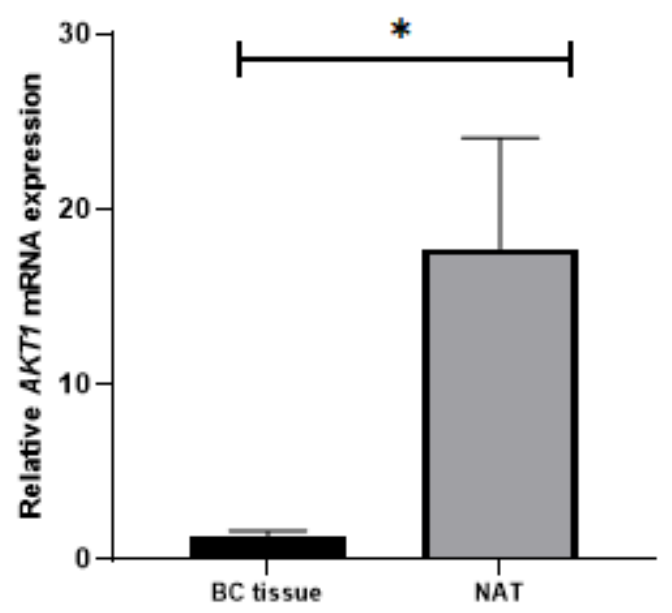

Figure 2

AKT1 mRNA expression is downregulated in BC tissues and NATs. Relative AKT1 expression levels in BC tissues and NATs were determined by qRT-PCR. The expression of $A K T 1$ is downregulated in BC tissues compared with NATs (* $p$ 0.05). GAPDH was used as an internal control. Abbreviation: BC: breast cancer, NATs: paired normal adjacent tissues.

a

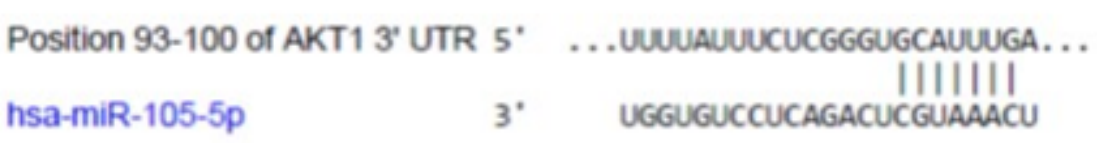

b

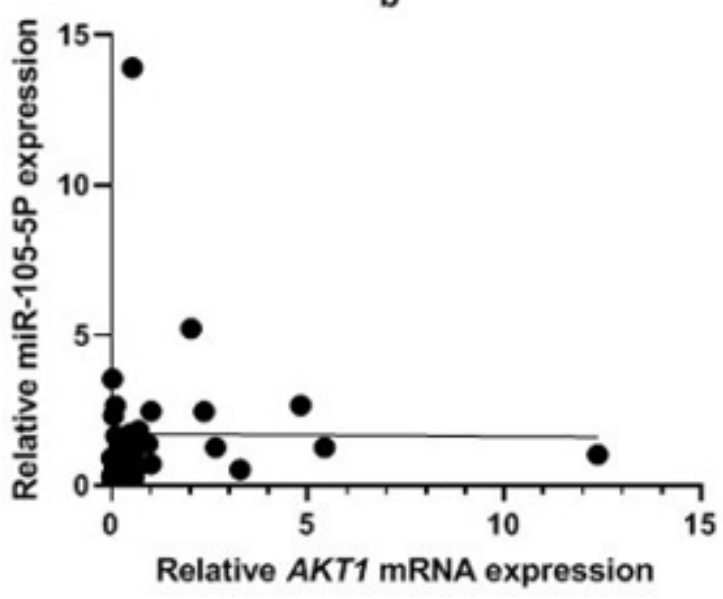

Figure 3 
AKT1 is a direct target of miR-105-5p in breast cancer. a) miR-105-5p and its putative binding sequence sites in the AKT1 3'-UTR. b) miR-105-5p expression has negative correlation with AKT1 expression in BC tissues, (slope $=-0.02466, \mathrm{P}=0.7569)$.

a

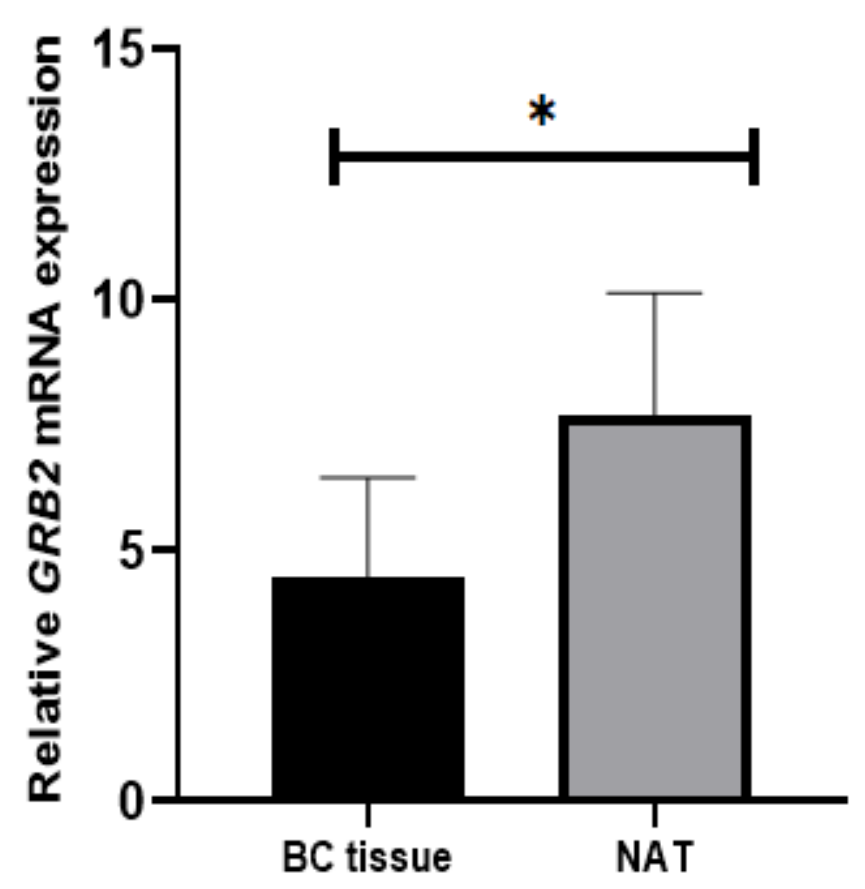

Figure 4

GRB2 mRNA expression is downregulated in BC tissues and NATs. Relative GRB2 expression levels in BC tissues and NATs were determined by qRT-PCR. The expression of miR-105-5p is significantly downregulated in BC tissues compared with NATs (* $p$ 0.05). GAPDH was used as an internal control. Abbreviation: BC: breast cancer, NATs: paired normal adjacent tissues. 
miRNA $3^{\circ}$ ugguguccucagacucGUAAACu $5^{\text {. }}$

Target $5^{\circ}$ cauucuacaccuggaccauUuga $3^{\text {. }}$

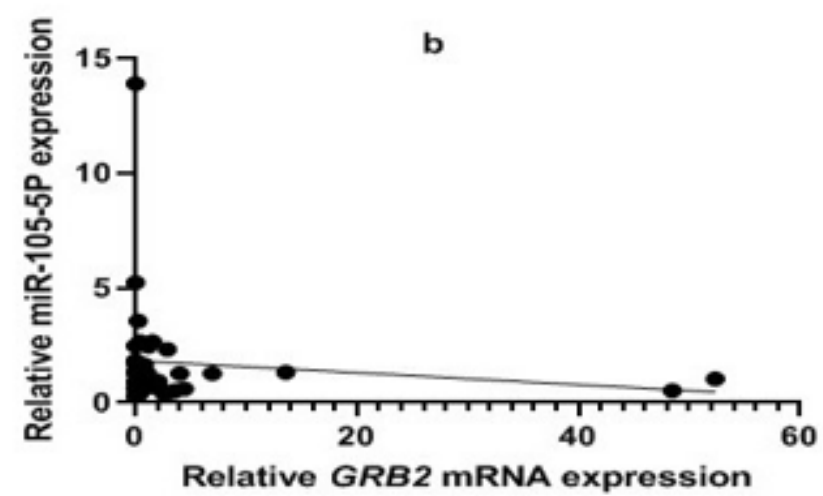

Figure 5

GRB2 is a direct target of miR-105-5p in breast cancer. a) miR-105-5p and its putative binding sequence sites in the GRB2 3'-UTR. miR-105-5p expression had negative correlation with the expression of GRB2 in $B C$ tissues, (slope $=-0.02098, p=0.1664)$ 